\title{
New Paramagnetic Susceptibility Thermometers for Fundamental Physics Measurements
}

\author{
D. A. Sergatskov ${ }^{1}$, P. K. Day ${ }^{2,3}$, A. V. Babkin ${ }^{1}$, R. C. Nelson ${ }^{4}$, T. D. McCarson ${ }^{1}$, \\ S. T. P. Boyd ${ }^{1}$, and R. V. Duncan ${ }^{1,3}$ \\ ${ }^{1}$ University of New Mexico, Albuquerque, NM 87131-1156 USA; ${ }^{2}$ Jet Propulsion Laboratory, Pasadena, CA \\ 91109-8099 USA; ${ }^{3}$ California Institute of Technology, Pasadena, CA 91125 USA; ${ }^{4}$ United States Military \\ Academy, West Point, NY 10996 USA
}

\begin{abstract}
New paramagnetic susceptibility thermometers have been developed for use in fundamental physics missions in earth orbit. These devices use a SQUID magnetometer to measure the variation in the dc magnetization of a thermometric element that consists of a dilute concentration of manganese in a palladium matrix. Near $2.2 \mathrm{~K}$ these new $\mathrm{PdMn}$ thermometers have demonstrated a temperature resolution of better than $100 \mathrm{pK} / \sqrt{\mathrm{Hz}}$ and a time constant of $50 \mathrm{~ms}$ when operated with a $50 \mathrm{~K} / \mathrm{W}$ thermal resistance to the liquid helium sample. These thermometers have been observed to be remarkably stable, with a drift of less than $10 \mathrm{fK} / \mathrm{s}$. The observed power spectral density of the noise from these thermometers is consistent with separate measurements of the device's time constant and thermal standoff from the bath. Recently these PdMn materials have been made into thin films and microstructures for use in future studies of quantum liquids, and for possible use in a new class of bolometers and radiometers. These thermometers have been integrated into an experimental cell and thermal isolation network that are adequate to keep stray heats stable to within a few picowatts, with no systematic temperature errors greater than $60 \mathrm{pK}$, over the course of a planned fundamental physics experiment on Earth orbit.
\end{abstract}

\section{INTRODUCTION}

We report on a new type of paramagnetic susceptibility thermometry that has been developed to facilitate upcoming fundamental physics experiments on low-earth orbit, including the Critical Dynamics in Microgravity Experiment (DYNAMX) [1], and the Enhanced Heat Capacity of Superfluid Helium in a Heat Flux Experiment (CQ) [2]. These devices have been used to explore new effects within quantum liquids on the ground, such as the elucidation of the self-organized heat-flow state [3], the mapping of the nonlinear heat-transport region [4], and the detection of new diffusion waves [5], all near the superfluid transition in ${ }^{4} \mathrm{He}$. A detailed report of these new devices, including a first-principles analysis of their noise performance, has been published previously [6]. Techniques were described at the end of Ref. [6] to improve the performance of these devices. Here we report on the experimental realization of these improvements, and we provide a guide to the construction of these devices. We also report on the development of new thin-film technology that will enable the use of these materials in new bolometer and high-bandwidth thermometer designs [7].

The thermometric element of these miniature $2.7 \mathrm{~g}$ thermometers consists of bulk $\mathrm{Pd}_{1-x} \mathrm{Mn}_{x}$ material with $x=6.8 \times 10^{-3}$. (For simplicity we refer to this material as PdMn below.) This thermometer design includes a niobium flux tube that traps a persistent magnetic field of about $50 \mathrm{G}$ within its interior, which is then incident on the PdMn element. A superconducting flux transformer wound around this PdMn is coupled to the input of a superconducting quantum interference device (SQUID), as discussed in Ref. [6]. This thermometric element is operated slightly above its Curie temperature, where the magnetic susceptibility of the PdMn is strongly temperature dependent. Hence small temperature changes result in changes in the flux threading the PdMn material, which in turn results in a proportional amount of flux change in the SQUID. Hence the output of the SQUID is calibrated against temperature using a primary thermometer, such as vapor pressure thermometry. Often a calibrated 
germanium resistance thermometer (GRT) is used as a transfer standard.

These devices have demonstrated a noise level of less than $100 \mathrm{pK} / \sqrt{\mathrm{Hz}}$, a rise-time constant of less than $20 \mathrm{~ms}$, and a drift rate of less than $10 \mathrm{fK} / \mathrm{s}$ (once the common platform drift was subtracted), all at temperatures near $2.2 \mathrm{~K}$. A brief summary of these results has been published earlier [8]. The source of noise in these thermometers has been accounted for in terms of fundamental noise sources, such as fluctuation-dissipation noise, thermally induced eddy current noise, and SQUID noise. As reported previously [6], these new devices display an enhanced sensitivity due to the itinerant electrons in the Pd that are correlated by the dilute $\mathrm{Mn}$ ions to create an exceptionally large effective magnetic moment, greatly enhancing the magnitude of the paramagnetic susceptibility of these materials [9].

This thermal performance, while impressive, is limited by our requirement that we couple to our sample through a $75 \mu \mathrm{m}$ thick foil in order to achieve the required spatial resolution on DYNAMX. This spatial resolution requirement results in a thermal stand-off from our sample of $50 \mathrm{~K} / \mathrm{W}$ due primarily to the thermal boundary resistance (called the Kapitza resistance) between the foil and the liquid helium. This thermal resistance may be greatly reduced in applications where the helium sample may directly wet the thermometric element. This, coupled with new

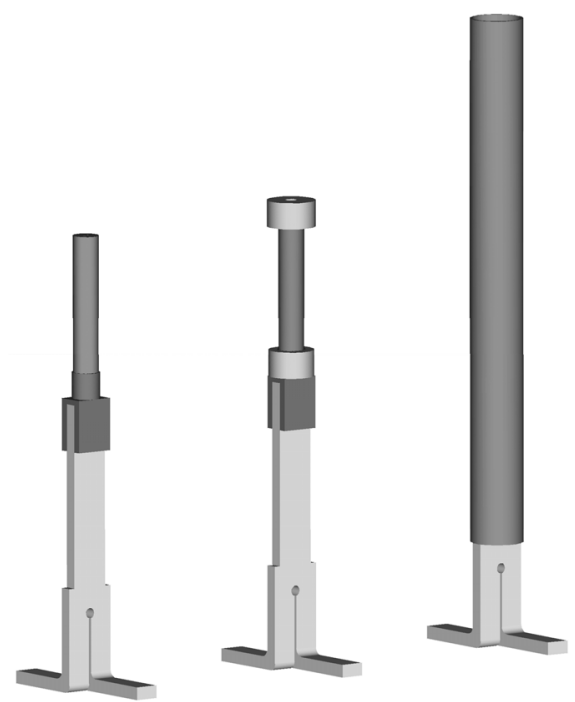

FIGURE 1. The assembly of the miniature high-resolution thermometer (HRT). The PdMn element is soldered to a copper fitting that is then diffusion welded to the pure aluminum foot. Ceramic spacers then guide the niobium flux tube in place. designs that reduce the mutual inductance between the current fluctuations in the thermometric element and the SQUID input loop, should result in future noise levels that are limited to less than $5 \mathrm{pK} / \sqrt{\mathrm{Hz}}$, which is set only by the flux noise level of the SQUID readout. New efforts are currently in process to achieve this level of thermometry resolution [10].

\section{THERMOMETER DESIGN, ASSEMBLY, AND PERFORMANCE}

As discussed by other authors [6, 11], the thermodynamic fluctuation noise of these paramagnetic susceptibility thermometers scales as $\sqrt{ } R$, where $R$ is the total thermal resistance (measured, for example, in kelvins per watt) between the PdMn thermometric element and the sample. This noise source is independent of the heat capacity of the PdMn, so the reduction of this noise source depends on minimizing this thermal resistance. Our sample in the DYNAMX and CQ experiments is liquid ${ }^{4} \mathrm{He}$, so the main contribution to this resistance in our flight experiments is the thermal boundary resistance, referred to as the Kapitza resistance, between our thermometry stages and the helium sample. All of the other resistances in series between the PdMn thermometric element and the sample have been greatly reduced from their corresponding values in earlier devices using the construction techniques described below. The assembly steps are illustrated in Fig. 1. A complete set of drawings and assembly procedures are available from our group, free of charge, upon request.

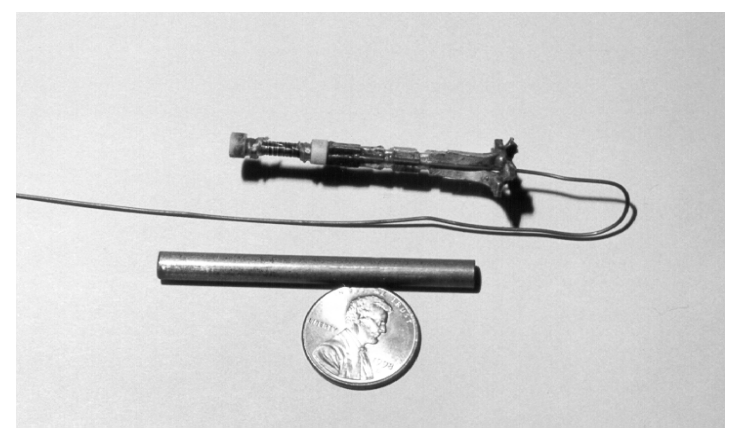

FIGURE 2. The assembled thermometer. The entire thermometer mass, including the flux tube, is less than three grams, and hence less than the mass of the penny.

These new PdMn materials have been fabricated by thoroughly mixing the correct ratio of a fine powder of $\mathrm{Pd}$ and $\mathrm{Mn}$, and then melting this mixture many times 
within an arc furnace, and then annealing [6]. The $\mathrm{PdMn}$ thermometric element is machined into a cylindrical shape and drilled along the axis. It is then brazed into a copper fixture in a tube furnace. The base of this copper fixture is then flattened in a press, and notched to fit around an aluminum post. The aluminum post is then diffusion welded into the notched copper fixture, and then ceramic spacers are placed at opposite ends of the PdMn. This assembled thermometer is displayed in Fig. 2. This manufacturing method provides a much more robust and superior thermal anchoring between the PdMn element and the sample stage than may be achieved in conventional designs that use paramagnetic salts, such as $\mathrm{Cu}\left(\mathrm{NH}_{4}\right)_{2} \mathrm{Br}_{4}+2 \mathrm{H}_{2} \mathrm{O}$ or $\mathrm{GdCl}_{3}$ as the thermometric element [12]. These electrically resistive and chemically corrosive salts must be grown onto an array of copper fingers or wires to achieve a much weaker thermal coupling than may be achieved in PdMn with this brazing technique. Superconducting wire is wrapped around the PdMn element to form the sensing coil that will be coupled to the SQUID input. This coil is wound to match the input inductance of the SQUID, which is about two microhenrys. These superconducting leads are threaded through a thin NbTi tube that acts as superconducting conduit. A niobium flux tube then forms the exoskeleton of the device, as displayed in Figures 1 and 2. This flux tube is used to trap a magnetic field of about $50 \mathrm{G}$ incident on the PdMn paramagnetic element when the tube transitions into its superconducting state under an external magnetic field, which is generated with an external electromagnet.

This miniature HRT has demonstrated a noise performance of $100 \mathrm{pK} / \mathrm{Hz}$. This may be seen in the low-frequency limit of the power spectral density (PSD) plot of the noise from this device displayed in Fig. 3. The rise and fall times of this thermometer were measured directly by applying a heat pulse to the base of the thermometer through a small surface mount heater. This $20 \mathrm{~ms}$ rise time differed from the $50 \mathrm{~ms}$ fall time displayed in Fig. 4 due to the delay in cooling of the device through its measured $50 \mathrm{~K} / \mathrm{W}$ resistance to the thermal bath. This observed $50 \mathrm{~ms}$ pulse relaxation time agreed well with the $10 \mathrm{~Hz}$ roll-off $(3 \mathrm{~dB})$ of the PSD. This is consistent with measurements of the heat capacity of PdMn of $1.0 \mathrm{~mJ} / \mathrm{gK}$ [13] and the total $1.0 \mathrm{~g}$ mass of this thermometric element.

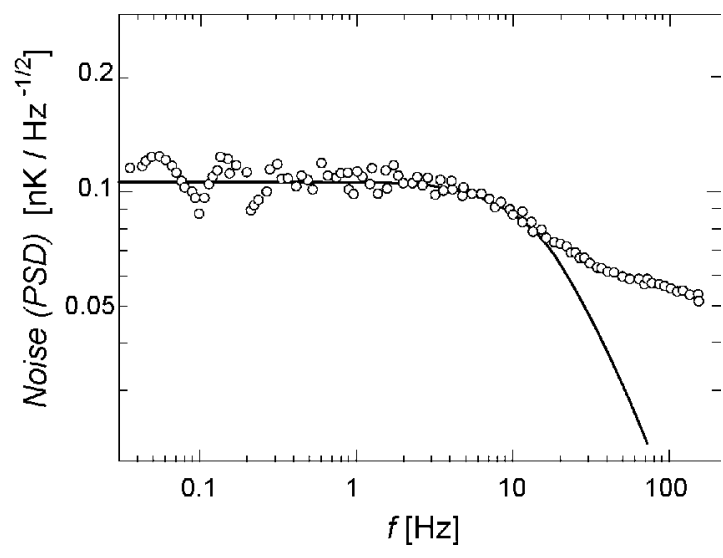

FIGURE 3. The power spectral density (PSD) of the noise from a PdMn thermometer.

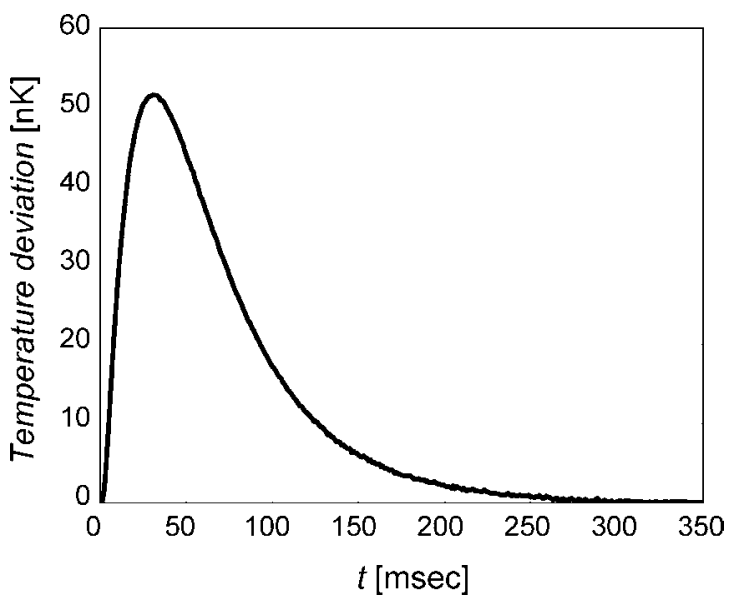

FIGURE 4. The response of the thermometer to a small heat pulse applied directly to the device. Notice that the rise time of $20 \mathrm{~ms}$ is much faster than the $50 \mathrm{~ms}$ relaxation time, since the heat applied to the thermometer directly had to cool to the liquid helium bath through a large thermal boundary (Kapitza) resistance.

\section{THIN FILM FABRICATION, DESIGN AND PERFORMANCE}

These materials have also been sputtered to form thin films and microstructures, and the temperature dependence of these devices has been measured as a function of temperature and ambient magnetic field [7]. These devices are useful in very high bandwidth applications, such as bolometry and high-speed thermometry. We find that relatively thick films (greater than $10 \mu \mathrm{m}$ ) may be used as thermometric 
elements in both their paramagnetic and ferromagnetic phases, while thin films (less than $1 \mu \mathrm{m}$ thick) are useful as non-hysteretic thermometers and bolometers only in their paramagnetic phase. A $10 \mu \mathrm{m}$ thick screen, consisting of $600 \mu \mathrm{m}$ diameter holes on $720 \mu \mathrm{m}$ centers to yield an effective open area of $70 \%$, has also been prepared using this sputtering technique on an ultrasonically milled sapphire substrate. These films are then separated from their substrate by repeated thermal cycling, permitting the free-standing microscreen to be used in applications where high spatial resolution, high bandwidth, and very low heat capacity are required. This screen may be operated with their charging magnetic filed perpendicular to the screen with a predictable decrease in sensitivity due to demagnetization, but still with adequate sensitivity to achieve a noise level of a few nanokelvins per root-hertz. This should prove adequate to observe the thermal distribution near heated boundaries in the superfluids, where the nature of the quantum order parameter's boundary condition may be explored experimentally through a careful measurement of the boundary thermal profile [14].

\section{CELL AND THERMAL ISOLATION NETWORK}

The thermometers discussed above are mounted with a liquid helium cell in the DYNAMX experiment as displayed in Fig. 5. Each of the three thermometers that connect to the cell sidewall foils are mechanically supported by the cell top endplate, but thermally isolated from this endplate through a thermally insulating tube. This construction results in more than a $10^{5} \mathrm{~K} / \mathrm{W}$ thermal resistance between each sidewall thermometer and the top cell plate. This cell and thermometer assembly is then mechanically supported by an annular cooling stage, as shown in Fig. 5. The cell assembly is cooled through the three structural supports between the cooling stage and the cell assembly, which collectively provide a thermal resistance between the cell and the cooling stage of about $2 \mathrm{kK} / \mathrm{W}$. In normal operation of the DYNAMX experiment the cell assembly remains within two microkelvins of the superfluid transition temperature $T_{\lambda}$ of the ${ }^{4} \mathrm{He}$ sample. The cooling stage temperature is controlled by another high resolution thermometer of similar design to a few millikelvins below $T_{\lambda}$, resulting in a heat extraction of a few microwatts of power from the cell through this $2 \mathrm{kK} / \mathrm{W}$ thermal resistance. This extracted heat is from electrical power dissipation on the heater resistors mounted on the cell endplates. The cooling stage temperature remains at its set point with a temperature noise of only two nanokelvins, resulting in a heat extraction noise of only about one picowatt. Since the cell temperature varies by no more than two microkelvins about $T_{\lambda}$ during the DYNAMX measurements, the total extracted heat from the cell remains constant to about $0.1 \%$ as the cell temperature varies over this very narrow experimental range.

The effects of any stray heating paths in the cell and support assembly were observed by maintaining the helium in its isothermal, superfluid state while the heat flux $Q$ through the cell was varied between zero and its maximum value. A control loop was used to adjust the heat dissipated in a resistor on top of the cell to maintain the cell temperature, as indicated by the top cell thermometer, to within $0.2 \mathrm{nK}$ of its set point value. The other two thermometers remained free running. All the thermometers displayed a transient associated with the control loop's response when $Q$ was changed abruptly. Once the transient died away all three thermometers reached their new steady state indication, which was then compared to the thermometer's readout before the change in $Q$. Since the superfluid remains isothermal under a heat flux, the extent to which the thermometers settle back to their original indication following a change in $Q$ provides a measure of the effect of any stray heat currents, and of any unintentional change in the control loop's set point. The maximum variation in all of the thermometer indications during a switch of $Q$ from zero to $100 \mathrm{nW} / \mathrm{cm}^{2}$ (the maximum value of $Q$ in the primary measurement range for the DYNAMX experiment) was $60 \mathrm{pK}$, as displayed in Fig. 6. This maximum change was about half as large as the random thermometry noise, indicating that this maximum systematic was of no major consequence, and it was easily small enough to meet the DYNAMX science requirement for flight. This resulted in a maximum systematic thermometry error with $Q$ of $0.06 \mathrm{nK} / 100 \mathrm{nW} / \mathrm{cm}^{2}=6 \times 10^{-4} \mathrm{~cm}^{2} \cdot \mathrm{K} / \mathrm{W}$. Since the CQ guest experiment requires a primary $Q$ range of $4 \mu \mathrm{W} / \mathrm{cm}^{2}$, the procedure above was repeated at twice this maximum on a later prototype of the same flight hardware design. The average free running thermometer error was $0.87 \mathrm{nK}$ after seven switches between zero and $8 \mu \mathrm{W} / \mathrm{cm}^{2}$. Hence the systematic thermometry error at this value of $Q$ was $1.1 \times 10^{-4} \mathrm{~cm}^{2} \cdot \mathrm{K} / \mathrm{W}$. 


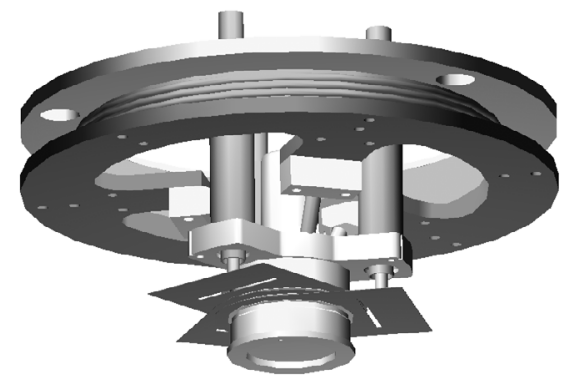

FIGURE 5. The cell and the cooling stage assembly. This assembly was surrounded by two radiation shields and maintained in a hard vacuum to minimize stray heat input. During the high temperature resolution tests the helium was maintained at its saturated vapor pressure by locating the liquid-vapor interface in a small attached chamber directly above the cell. For other details, see text.

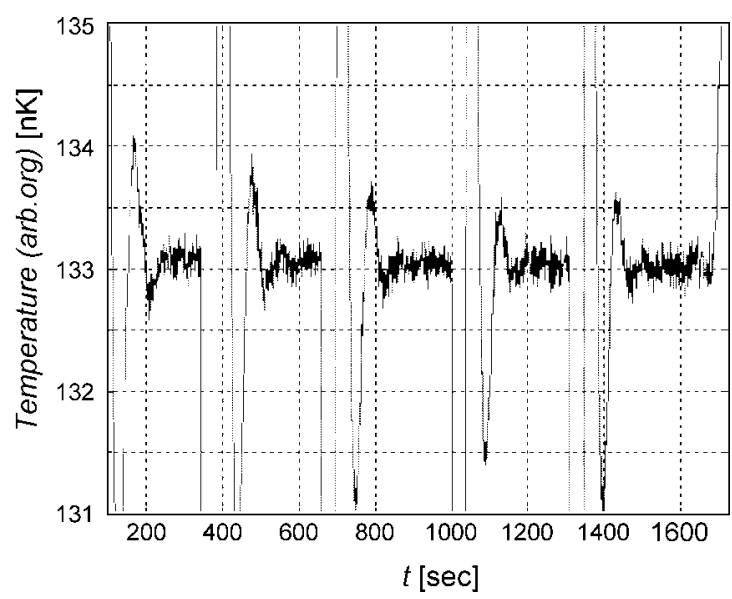

FIGURE 6. Experimental determination of the maximum systematic thermometry error displayed in the middle thermometer on the cell that was associated with a change of heat flux between zero and $100 \mathrm{nW} / \mathrm{cm}^{2}$, as described in the text. The maximum thermometry error under this change of $Q$ was $60 \mathrm{pK}$, resulting in a maximum systematic error of $6 \times 10^{-4} \mathrm{~cm}^{2} \cdot \mathrm{K} / \mathrm{W}$. Here $Q$ was first switched from $100 \mathrm{nW} / \mathrm{cm}^{2}$ to zero, and then back and forth two more times.

\section{CONCLUSIONS}

A new method of paramagnetic susceptibility thermometry has been developed based on dilute concentrations of manganese in palladium. These materials have resulted in exceptionally high resolution thermometers with very low measured drift rates. These materials are stable metallic alloys which have been sputtered to produce thin films for fast thermometry and Bolometry applications.

\section{ACKNOWLEDGMENTS}

This work has been supported through the Fundamental Physics Discipline of the Microgravity Science Office of the United States National Aeronautics and Space Administration (NASA) under JPL Contract Number 960494. One of us (R.C.N.) acknowledges support from the United States Army.

\section{REFERENCES}

1. NASA Science Requirements Document for "Critical Dynamics in Microgravity", JPL Document Number D18698, May 31, 2000.

2. NASA Science Requirements Document for "Enhanced Heat Capacity of Superfluid Helium in a Heat Flux" to become a JPL Document.

3. Moeur, W. A., Day, P. K., Liu, F-C., Boyd, S. T. P., Adriaans, M. J., and Duncan, R. V., Phys. Rev. Lett. 78 2421-2424 (1997).

4. Day, P.K., Moeur, W.A., McCready, S.S., Sergatskov, D. A., and Duncan, R. V., Phys. Rev. Lett. 81, 2474-2477 (1998).

5. Sergatskov, D. A., Babkin, A. V., Lee, R. A. M., Boyd, S. T. P., and Duncan, R. V., Proceedings of LT23, to appear in Physic (2002).

6. Klemme, B. J., Adriaans, M. J., Day, P. K., Sergatskov, D. A., Aselage, T. L., and Duncan, R. V., J. Low Temp. Phys. 116, 133-146 (1999).

7. Nelson, R. C., Sergatskov, D. A., and Duncan, R. V., J. Low Temp. Phys. 127, 173-188 (2002).

8. Duncan, R. V., Sergatskov, D.A., Babkin, A. V., Boyd, S. T. P., Nelson, R. C., McCarson, T. D., Day, P. K., Dooley, J. A., and Elliott, D. G., Second Pan Pacific Basin Workshop on Microgravity Sciences, Paper FP1067 (2001).

9. Star, W. M., Foner, S., and McNiff, E. J., Phys. Rev. B 12, 2690 (1975).

10. Lee, C., Sergatskov, D. A., and Duncan, R.V., to be published.

11. Day, P. K., Hahn, I., and Chui, T. C. P., J. Low Temp. Phys. 107, 359 (1997).

12. Lipa, J. A., Leslie, B. C., and Wallstrom, T. C., Physica B 107331 (1981).

13. Boerstoel, B.M., Zwart, J. J., and Hansen, J., Physica 57, 397 (1972).

14. Nelson, R. C., Development of Thin-Film PdMn High Resolution Thermometry for Measurement of the $4 \mathrm{He}$ Superfluid Boundary Layer, Ph.D. Thesis, U. New Mexico (2001). 